\title{
INTRODUCCIÓN
}

\section{¿Por qué Funcionan los Tratamientos Psicológicos?}

\section{Why do Psychological Treatments Work?}

\author{
María Xesús Froján Parga \\ Universidad Autónoma de Madrid
}

Por qué funcionan los tratamientos psicológicos o, dicho de otra manera, cuáles son los procesos que subyacen o explican el cambio en terapia, son preguntas que cualquier psicólogo en general y el clínico en particular, está interesado en contestar. Sin embargo la respuesta no es fácil, a pesar de los muchos intentos que se han realizado en este sentido. En este número tenemos una muestra de ellos, planteados por algunos de los principales autores en cada área. Veremos perspectivas diversas, con algunas podemos estar de acuerdo y con otras no, pero que siempre son fruto de la reflexión y/o experimentación que deben acompañar al progreso de cualquier ciencia.

Tradicionalmente, en el campo clínico han coexistido dos grandes líneas de investigación que abordan el cambio terapéutico de manera distinta y, desde nuestro punto de vista, complementaria: la investigación de resultados (¿qué es mejor que qué?), dominante en el campo hasta el momento actual y que ha culminado con la especificación de la práctica psicológica basada en la evidencia; y la investigación de procesos (¿cómo y por qué funciona lo que funciona?), olvidada durante años y que a partir de la década de los 90 empezó a resurgir con fuerza, dando lugar a algunos de los trabajos más interesantes que ha habido en estos últimos años en el tema que nos ocupa. Es interesante saber qué tratamientos son mejores que otros pero, desde nuestra perspectiva, todavía lo es más saber qué los hace mejores. Conocer por qué funcionan los tratamientos psicológicos y qué procesos explican el cambio clínico permitirá un desarrollo verdaderamente sólido de la intervención en psicoterapia.

La investigación realizada sobre el proceso terapéutico nos lleva a destacar cinco pilares fundamentales de la terapia psicológica:
1. La reinvidicación de un modelo terapéutico esencialmente psicológico, con una herramienta única e insustituible que es el análisis funcional. Atendiendo a la funcionalidad de la conducta del cliente podemos explicar los problemas que lo traen a consulta y podemos diseñar y aplicar técnicas de intervención que permitan desarrollar nuevas funcionalidades más adaptativas a largo plazo. Los psicólogos contamos con una estrategia de análisis y tratamiento que caracterizan un modelo propio y sitúa el proceso terapéutico en un plano radicalmente distinto del de otros profesionales con los que supuestamente pueden estar en conflicto. Ya no se trataría, por tanto, de diferenciarnos en virtud de una estrategia de intervención concreta o de destacar la discutible base orgánica de determinadas patologías sino en el planteamiento de un modelo de explicación y tratamiento que abarcaría la totalidad de la conducta humana. Y utilizamos el término conducta con toda la amplitud que permite, esto es, como interacción entre una respuesta (encubierta o manifiesta, cognitiva o motora, verbal o emocional) y el contexto en el cual se da. La conducta, por tanto, no sería acción ni reacción, sino interacción.

2. El análisis de la conducta verbal durante la interacción terapéutica. Aunque la terapia es fundamentalmente hablada, hasta muy recientemente no se ha planteado un estudio formal de la interacción verbal entre el psicólogo y el cliente y una conceptualización de lo que se dice en terapia como una conducta clínicamente relevante (en el sentido planteado por la Psicoterapia Analítico Funcional y presentada por Valero-Aguayo, Ferro-García, Kohlenberg y Tsai en este mismo número).

3. La consideración de la situación clínica como un contexto natural en el que se dan los problemas 
en la misma forma en que pueden aparecer en el contexto extra-terapéutico; ya no sólo es que cambiando lo que una persona dice sobre lo que hace se puede cambiar lo que hace sino que al "comportarse" en terapia una persona puede mostrar los mismos problemas que la hacen buscar ayuda psicológica, exactamente igual que se mostrarían en cualquier otro contexto social. En este sentido, la situación terapéutica se constituiría en un contexto único donde establecer contingencias de reforzamiento y de castigo sobre las propias conductas objetivo de tratamiento.

4. El estudio de la relación terapéutica como un proceso interactivo, fruto del cual se va desarrollando una relación que será terapéutica en sí misma (en el sentido de que dará lugar a esos procesos de aprendizaje que son, en último término, los responsables del cambio clínico). En este monográfico, Krause, Altimir y Horvath abordan un concepto clave en el proceso terapéutico que es el de alianza; tradicionalmente, este concepto (como muchos otros que se han utilizado de forma equivalente) se ha analizado desde una perspectiva estática, atendiendo a variables del cliente y del terapeuta que podían favorecer el establecimiento de una buena relación en terapia y que eran ajenos a la propia intervención. Sin embargo, desde nuestra perspectiva, consideramos que el establecimiento de una buena relación o alianza terapéutica depende del proceso interactivo que tiene lugar durante la sesión de terapia; en este sentido, la relación o alianza sería fruto, consecuencia, de la interacción terapéutica y haciendo que esta interacción tenga unas características determinadas (específicas para cada caso) se conseguirá el establecimiento de una buena alianza. De esta manera dejaría de tener sentido la antigua polémica acerca de qué pesa más, si la relación terapéutica o las técnicas de intervención en la explicación del cambio clínico, puesto que una y las otras serían la ocasión para la ocurrencia de los procesos de aprendizaje.

5. La conducta encubierta (cognitiva) tiene las mismas características y puede ser abordada de la misma forma que la conducta manifiesta; el hecho de que sea encubierta no cambia la cualidad de la conducta, ni la hace inobservable ya que, al menos, es siempre observable por un observador (uno mismo). Y ese observador la puede hacer pública a través (aunque no exclusivamente) de sus verbalizaciones; no queremos entrar aquí en la polémica acerca de si lo cognitivo es igual o diferente de lo verbal (o si pensar es un hablar silente, utilizando los términos de Marino Pérez en su obra Contingencia y drama), pero de lo que consideramos que no hay duda es de que, en terapia, el procedimiento principal para tener acceso a las cogniciones del cliente es precisamente lo que nos dice sobre lo que piensa. La famosa técnica de reestructuración cognitiva, un clásico en los manuales del enfoque cognitivo-conductual (la cursiva quiere destacar lo redundante del término) únicamente trabaja con verbalizaciones que se van modificando a través del debate socrático para, en último término, concluir que como fruto del cambio en las verbalizaciones han cambiado las cogniciones.

¿Qué sería, por tanto, lo que hace el psicólogo cuando hace terapia? Desde nuestra perspectiva, lo que hace es poner en marcha (o al menos intentarlo) una serie de procesos de aprendizaje (tanto pavlovianos como operantes) que darán lugar a la aparición de nuevas conductas, más adaptativas, menos problemáticas, en el cliente. Y estos procesos de aprendizaje ocurren a través (aunque no exclusivamente) de la interacción verbal que tiene lugar durante la sesión terapéutica. De esta conceptualización de la actuación clínica como desarrollo de procesos de aprendizaje que ocurren en el contexto terapéutico han surgido las terapias contextuales que han renovado el panorama de la intervención conductual en las dos últimas décadas. En última instancia, el cambio de las verbalizaciones del cliente en sesión ha de ser utilizado para promover y mantener los cambios extra-clínicos, de manera que las nuevas verbalizaciones discriminen conductas más adaptativas en la vida cotidiana, tal como plantean Schlinger y Alessi por un lado y Salzinger, por otro, en sus respectivos artículos de este monográfico.

Pero aún podemos ir más allá en nuestra conceptualización del proceso terapéutico y podemos extender ésta a cualquier tipo de intervención, se adscriba al modelo que se adscriba. Quiere esto decir que los procesos de aprendizaje ocurren, los conozcamos o no, los queramos provocar con la aplicación de técnicas concretas o surjan de manera espontánea. Si el 
psicólogo los conoce, la probabilidad de que dichos procesos ocurran y den lugar a los cambios en la dirección deseada será mayor y la intervención será más eficaz; si, por el contrario, el clínico los desconoce, los procesos se darán igualmente, aunque quizás con más demora o con menor control por parte del psicólogo. Los procesos de aprendizaje son al cambio clínico lo que la electricidad es a la iluminación de una habitación: podemos tener diferentes tipos de lámparas, de interruptores o de bombillas, pero lo que hace que haya luz es la electricidad. La forma en la que ésta se haga patente (una lámpara de araña o una simple bombilla) es secundaria al circuito que hace llegar la corriente eléctrica desde el interruptor a la fuente de iluminación. Si conocemos algo de electricidad, nuestro circuito será más eficaz pero si no lo conocemos, probaremos diversas conexiones hasta que al final se haga la luz.

Hay una última cuestión que nos gustaría comentar en nuestra breve introducción a este monográfico: la consideración del lenguaje como el mecanismo que permite traer el presente hechos pretéritos, que de esta forma adquieren una funcionalidad actual. Quiere esto decir que pierde todo sentido la vieja distinción entre pasado y presente a la hora de analizar un problema clínico o la insistencia en rechazar el modelo conductual porque "olvidaba" aspectos de la problemática de las personas (sus vivencias pasadas) que podían ser fundamentales para entender lo que ocurría; en el plano psicológico, pasado y presente forman parte de un único conjunto que puede ser funcionalmente importante: cuando un cliente habla de sus experiencias pasadas (de motu propio o "forzado" por la propia intervención terapéutica), su discurso hace presentes tales experiencias, de manera que el papel que tengan en el momento actual lo tienen porque es presente (al hablarlo, sentirlo o comportarse con una morfología determinada). Si algo ocurrió en el pasado, por grave o relevante que haya sido en su momento, que no está funcionalmente relacionado con el comportamiento actual (patológico o no, problemático o saludable) de una persona, ese algo pasado no contribuye a explicar éste; y por el contrario, un acontecimiento pretérito irrelevante puede adquirir una importancia clave en la explicación del problema actual cuando al traerlo al presente por medio del lenguaje podemos identificar su funcionalidad. La historia de aprendizaje de una persona es presente desde el momento que tiene un valor funcional sobre comportamientos actuales y esta funcionalidad se puede estudiar a partir de las verbalizaciones que dicha persona da cuando describe las relaciones que ha establecido entre diversos eventos ocurridos a lo largo de su vida. Estas relaciones son "presente", aunque describan hechos pasados.

Todas estas cuestiones que hemos resumido aquí en torno al proceso de cambio en terapia podemos encontrarlas ampliamente desarrolladas en los artículos de este número. Los autores han destacado aquellos aspectos que consideran importantes para explicar el cambio terapéutico. Caro presenta el proceso clínico a la luz del modelo de asimilación de experiencias problemáticas, concluyendo que la asimilación se relaciona con cambio terapéutico mientras que la no asimilación se asocia a fracaso. Por su parte, Krause, Altimir y Horvath clarifican el concepto de alianza terapéutica y evalúan la calidad de la misma en función de cómo atienden clientes y terapeutas a distintos aspectos de la relación. Schlinger y Alessi plantean el estudio de los cambios en la conducta del cliente a través de las interacciones verbales que tienen lugar en el contexto clínico; estos autores explican la generalización del cambio a los contextos habituales del cliente a partir de los procesos de condicionamiento verbal en la situación de terapia, que tienen el poder de alterar la función de los estímulos presentes en los contextos extra-clínicos y dar lugar a conductas diferentes (más sanas). El artículo de Salzinger defiende la utilización de un único modelo científico para explicar cualquier intervención terapéutica, destacando la importancia del condicionamiento operante. En la misma línea, Valero-Aguayo, Ferro-García, Kohlenberg y Tsai destacan el tronco común de las terapias de tercera generación, que estaría constituído por los estudios de relaciones de equivalencia entre estímulos, el análisis funcional del lenguaje y su repercusión sobre la conducta emocional y cognitiva; su plantemiento del contexto terapéutico como un contexto natural funcionalmente similar a la vida cotidiana es la base de la Psicoterapia Analítica Funcional. Por último, Tonneau cuestiona la base de la terapia cognitiva y propone un análisis experi- 
mental de los conceptos que se plantean, insistiendo en que el modelo operante no es suficiente para explicar el cambio clínico y reivindicando el papel de los procesos pavlovianos.

Para finalizar esta introducción, queremos agradecer desde aquí la participación de los autores de este monográfico que han posibilitado la presenta- ción de un completo panorama de los procesos explicativos del cambio clínico, que esperamos que sirva de reflexión y análisis para todas aquellas personas que trabajamos en el área y que, a pesar de las muchas y acusadas diferencias que podemos encontrar, tenemos un objetivo común: ayudar a nuestros clientes a ser más felices. 